\title{
A UNIFIED APPROACH TO MODELING MULTIDISCIPLINARY INTERACTIONS
}

\author{
Jamshid A. Samareh* \\ NASA Langley Research Center \\ Hampton, VA \\ Kumar G. Bhatia ${ }^{\dagger}$ \\ The Boeing Company \\ Seattle, WA
}

\begin{abstract}
$\underline{\text { Abstract }}$
There are a number of existing methods to transfer information among various disciplines. For a multidisciplinary application with $n$ disciplines, the traditional methods may be required to model $\left(n^{2}-n\right)$ interactions. This paper presents a unified three-dimensional approach that reduces the number of interactions from $\left(n^{2}-n\right)$ to $2 n$ by using a computer-aided design model. The proposed modeling approach unifies the interactions among various disciplines. The approach is independent of specific discipline implementation, and a number of existing methods can be reformulated in the context of the proposed unified approach. This paper provides an overview of the proposed unified approach and reformulations for two existing methods. The unified approach is specially tailored for application environments where the geometry is created and managed through a computer-aided design system. Results are presented for a blended-wing body and a high-speed civil transport.
\end{abstract}

\section{Introduction}

A key element in the application of multidisciplinary design optimization (MDO) to an engineering system is the introduction of a consistent geometric representation. Such a representation guarantees that the same geometry model is used to derive the computational models required for various disciplinary analyses. By utilizing computeraided design (CAD) for consistent geometry representation, it is easier to analyze complex configurations with higher-fidelity tools such as computational fluid dynamics (CFD), computational structural mechanics (CSM), or detailed finite-element analysis.

A feature that characterizes multidisciplinary analysis and optimization is the modeling of interactions among various disciplines. For example, the strong interactions between CSM and CFD can prompt physically important phenomena such as those occurring in aircraft due to aeroelasticity. Correct modeling of these complex aeroelastic phenomena requires a coupling of CSM and CFD for a flexible structure (e.g., airplane). In a multidisciplinary environment, various disciplines must represent the same configuration geometry, and data from each discipline must be available consistently to all the disciplines. The data may be scalar (e.g., pressure and temperature), vector (e.g., deflection and heat transfer), or integrated quantities (e.g., aerodynamic and thermal loads). The data transfer process may be subjected to additional constraints, such as conservation of forces, moments, and energy. The focus of this paper is the transfer of data between dissimilar grids (most models do not share the same nodal locations at the interface).

\footnotetext{
- Research Scientist, Multidisciplinary Optimization Branch, AIAA Senior Member

${ }^{\dagger}$ Senior Technical Fellow, Aeroelasticity and Optimization, AIAA Associate Fellow
} 


\section{Review of Existing Methods}

The issue of exchange of pressure distribution and displacements between an aerodynamic model and a structural model gained urgency with the first widespread use of panel aerodynamic methods and finite-element structural models during the late sixties. The traditional beam and strip theory methods were not applicable to the large, flexible low-aspect ratio configuration of a supersonic transport (SST). The SST experience led to development of a comprehensive aeroelastic computer program called FLEXSTAB. ${ }^{1}$ Most of the work was done under NASA funding before 1974. The basic theoretical work was completed between 1968-71, and the goal of FLEXSTAB was to provide an aeroelastic program for production use. FLEXSTAB probably was the first computing system to address systematically all the aeroelastic transfer issues for a complete airplane configuration. FLEXSTAB was successful in providing an acceptable method for the pressure and displacement transfers. However, the method was time consuming, and required significant manual effort for the user to establish direct correspondence between the aerodynamic panels and structural elements

In the eighties Dassault Aviation developed a more simplified method ${ }^{2,3}$ including many practical considerations. The Dassault method is embodied in Elfini and includes several innovative ideas in aeroelastic formulation. First, Dassault used an intermediate computational grid between an aerodynamic mesh and a structural finite-element model for a given configuration. Thus different aerodynamic models could be used with the same structural model, and vice versa. Second, they used shape functions defined on the computational grid, and smoothing operators to relate structural displacements to the aerodynamic mesh. Third, they introduced the idea of loads basis on the computational grid (unit loads at the nodes of the computational grids) to transfer pressure from the aerodynamic mesh to the computational grid, and from the computational grid to the finite-element nodes. The overall approach is elegant and practical. But it still requires significant user input (although much less than FLEXSTAB) and checking to ensure the accuracy of the transfers.
In the recent years, various researchers have examined the issue of aeroelastic transfer. Discrepancies and dissimilarities in geometry and grid models are two potential sources of error. The accuracy of the data transfer depends on the relative resolutions of disciplinary grids. Data could be lost in transfer from a coarse grid to a fine grid. Another source of error occurs if the models have dissimilar levels of geometry detail. As noted by Tzong et al., ${ }^{4}$ a CFD grid generally resembles the true geometry of the aircraft; the grid includes details such as pylons, nacelles, flaps, and slats. However, a CSM grid generally represents only major structural components, such as the wing box. Flaps and slats are represented either by a few simple beam elements or are completely excluded. Tzong et al. ${ }^{4}$ and Kapania and Bhardwaj ${ }^{5}$ have developed methods, based on finite element (FE) technology, in which virtual work is employed to transfer the aerodynamic pressures onto a CSM grid. The displacements are then converted back to a CFD grid through the reciprocal theorem. Kapania and Bhardwaj ${ }^{5}$ were successful in using a simplified version of this method for several wings.

Brown ${ }^{6}$ added virtual elements in the CSM model to cover the discrepancies in the geometry definition between CSM and CFD models. These virtual elements add neither stiffness nor mass. As pointed out by Cebral and Löhner, ${ }^{7}$ the generation of the virtual elements is an unnecessary complication, particularly for complex geometries.

Hounjet and Meijer ${ }^{8}$ and Smith, Hodges, and Cesnik ${ }^{9}$ provided overviews of the data transfer methods. Smith, Hodges, and Cesnik evaluated six methods for transferring information between CFD and CSM disciplines. These methods were infinite-plate spline (IPS), multiquadric biharmonic (MQ), nonuniform B-spline (NUBS), thin-plate spline (TPS), finite-plate spline (FPS), and inverse isoparametric mapping (IIM). These methods have been implemented in a single code, FASIT ${ }^{10}$. The IPS method is based on the popular surface splines ${ }^{11}$ and is implemented in some commercial aeroelastic analysis tools. This method is designed for interpolating a function of two variables.

Out of these six methods, Smith, Hodges, and Cesnik ${ }^{9}$ recommended further study of $I \mathrm{M}$ and NUBS. They indicated that IIM shows great promise for two-dimensional applications and 
needed to be extended to three dimensions. Clutter $^{12}$ and Send ${ }^{13}$ extended NUBS to three dimensions.

One problem found with the NUBS implementation is that the data must be input as a structured (regular) grid. This requirement forces the data, at best, to be approximated, and in most realistic cases, this step is either time consuming or impossible. Samareh ${ }^{14}$ proposed a method to use non-uniform rational B-spline (NURBS) representation for data transfer among various disciplines. Because this method is based on a general three-dimensional, leastsquares representation, ${ }^{15.16}$ it does not require the input to be a structured grid. Another advantage of this approach is control over the tradeoff between smoothness and accuracy.

The accuracy of the data transfer process for integrated quantities (e.g., forces, moments, and energy) depends on the consistency of data transfer as well as other constraints, such as conservation. For example, a consistent load vector for CSM is defined as

$$
\left\{\bar{f}_{e}\right\}=\int_{S}\{N\}^{T} \bar{n}\{P\} d S
$$

where $\left\{\bar{f}_{e}\right\}$ is the element load vector, $\{N\}$ is the FE shape function, $\bar{n}$ is the unit surface normal, $P$ is the pressure, and $d S$ is the infinitesimal surface element.

Because the above equation uses the same shape functions as are used to calculate the element stiffness matrix, the equation guarantees a consistent loading or lumping. Cook, Malkus, and Plesha ${ }^{17}$ provided a detailed description of this equation. There are several possible problems with using the above equation. First, the aerodynamic load may have a large variation within a single $F E$, such that the shape function is not adequate to capture the variation. Second, the FE shape function may not be available for some commercial CSM codes. Third, in its present form the above equation does not guarantee conservation of forces and moments.

Maman and Farhat $^{18}$ outlined a consistent interpolation-based algorithm (IBA) for transferring information between two dissimilar grids. The algorithm is similar to the IIM proposed by Murti $^{19}$ for a two-dimensional model; it uses the FE shape functions to interpolate the coordinates, pressure, and displacement vectors. The method does not require any matrix inversion. Because the FE shape functions satisfy a positivity constraint, the process will not create nonexisting local extrema. The local interpolation is computed by projecting one grid onto another. Cebral and Löhner ${ }^{7.20}$ presented a variation of the IBA that could guarantee the conservation of forces. They used a Galerkin method to solve for pressure from the CSM grid. The Cebral-Löhner method requires a matrix inversion. They also used an adaptive Gaussian integration technique to improve the accuracy. Farhat, Lesoinne, and LeTallec ${ }^{21}$ also presented a variation of the original IBA ${ }^{18}$ that can guarantee conservation of forces, but requires no matrix inversion. We used this algorithm for the current study.

\section{Unified Approach}

\section{Overview}

The unified approach has two essential ingredients. First, the data transfer process between two disciplines was modeled by a transformation matrix. Second, the CAD model was used to reduce the number of interactions.

The interaction between two disciplines is modeled mathematically as

$$
\left\{F_{2}\right\}=\left[T_{21}\right]\left\{F_{1}\right\}
$$

where matrices $\left\{F_{1}\right\}$ and $\left\{F_{2}\right\}$ contain the information on discipline grids 1 and 2 , respectively, and matrix $\left[T_{21}\right]$ is a transformation matrix. For example, $\left\{F_{1}\right\}$ could be an aerodynamic loads vector defined on a CFD grid and transferred to the CSM grid as $\left\{F_{2}\right\}$. Generally the transformation matrices are sparse and large. Only the nonzero elements need to be stored. If the transformation matrix $\left[T_{21}\right]$ is independent of the shape changes, then $\left[T_{21}\right]$ can be calculated once and used as long as there is no change in the grid connectivity.

The concept of a transformation matrix simplifies 
integrated analyses such as aeroelastic calculation. The aeroelastic calculation has four distinct steps. First the aerodynamic loads are calculated on the CFD grid. Second the loads are transferred to the CSM grid. Third the aeroelastic deflections are calculated on the CSM grid. Fourth the deflections are transferred to the aerodynamic grid to recalculate the aerodynamic loads. This iterative process can be expressed as

$$
\begin{gathered}
\left\{\bar{F}_{\mathrm{F}}\right\}=\left\{\text { Flow Solution }\left(\bar{G}_{F}+\bar{\delta}_{\mathrm{F}}\right)\right\} \\
\left\{F_{S}\right\}=\left[T_{S F}\right]\left\{F_{F}\right\} \\
{[K]\left\{\bar{\delta}_{\mathrm{S}}\right\}=\left\{\bar{F}_{\mathrm{S}}\right\}} \\
\left\{\bar{\delta}_{\mathrm{F}}\right\}=\left[T_{F S}\right]\left\{\bar{\delta}_{S}\right\}
\end{gathered}
$$

The first equation represents the aerodynamic load calculation. The term $\bar{G}_{F}$ represents the CFD grid, and $\bar{\delta}_{\mathrm{F}}$ is the aeroelastic deflection on the CFD grid. The aerodynamic forces, $\bar{F}_{\mathrm{F}}$, are transferred to the CSM grid as $\bar{F}_{S}$. The matrix $[K]$ is the CSM stiffness matrix, and $\bar{\delta}_{\mathrm{S}}$ is the aeroelastic deflection on the CSM grid.

The use of the transformation matrix simplifies the above set of equations to

$$
\begin{gathered}
{[K]\left\{\bar{\delta}_{\mathrm{S}}\right\}=\left[T_{S F}\right]\left\{\bar{F}_{\mathrm{F}}\right\}} \\
\left\{\bar{F}_{\mathrm{F}}\right\}=\left\{\bar{F}_{\mathrm{F}}\left(\bar{G}_{F}+\left[T_{F S}\right]\left\{\bar{\delta}_{\mathrm{S}}\right\}\right)\right\}
\end{gathered}
$$

For a linear structure without rigid body degreesof-freedom, the above equation set can be simplified to

$$
\begin{gathered}
\left\{\bar{\delta}_{\mathrm{F}}\right\}=\left[T_{F F}\right]\left\{\bar{F}_{\mathrm{F}}\right\}, \text { where } \\
{\left[T_{F F}\right]=\left[T_{F S} \| K\right]^{-1}\left[T_{S F}\right]}
\end{gathered}
$$

The formulation can be extended to the case with rigid body degrees-of-freedom, and it allows decoupling of the CFD code from a linear CSM code.

If the transformation matrix is independent of the shape changes, then the formulation is especially beneficial. The benefit results from the fact that the sensitivity derivatives used in gradient-based optimization with respect to a vector of shape design variables $\left\{V_{i}\right\}$ will not require the differentiation of the transformation matrix. For example, the following relation could transfer the sensitivity of the CFD load to the CSM grid:

$$
\frac{\partial \bar{F}_{\mathrm{S}}}{\partial V_{i}}=\left[T_{\mathrm{SF}}\right] \frac{\partial \bar{F}_{\mathrm{F}}}{\partial V_{i}}
$$

For the six methods described in Ref. 9, the transformation matrices are dependent directly on the shape changes. However, NURBS and IIM methods can be reformulated to result in methods with transformation matrices that are independent of the shape changes. Samareh ${ }^{14}$ proposed a reformulation for NURBS, and Maman and Farhat $^{18}$ proposed a general alternative approach for IIM. This paper provides reformulations for both the methods.

The second ingredient of the proposed unified approach helps to reduce the number of transformation matrices. For a multidisciplinary application that involves $n$ disciplines, the traditional process may require $\left(n^{2}-n\right)$ transformation matrices. However, some of these couplings are either weak or nonexistent. Figure 1 shows all possible interactions among eight disciplines; modeling all interaction requires 56 transformation matrices.

The problem can be further complicated for a variable-fidelity multidisciplinary application. For example, aerodynamic loads can come from wind tunnel databases, or from linear aerodynamics, potential flow, Euler, and NavierStokes analysis codes. The following section provides details of an approach where the number of couplings is reduced from $\left(n^{2}-n\right)$ to $2 n$ by incorporating a CAD model.

\section{CAD-Based Approach}

The new element of the CAD-based approach was the use of a CAD geometry representation to reduce the number of transformations from $\left(n^{2}-n\right)$ to $2 n$. The reduction is accomplished by transferring the data to a CAD geometry 
model that serves as a common bridge or a data bus for sharing information among various disciplines, as shown in the Fig. 2.

This approach is of obvious benefit for multidisciplinary applications with more than three disciplines. However, the method offers benefits even when only two disciplines are involved. The intermediate CAD grid isolates each discipline model from changes to all other discipline models. The overall approach has a strong potential for robust automation of all transformations, and ensures consistency among different renderings of the same configuration.

First the data is transferred from the individual source discipline to the CAD model as

$$
\left\{F_{\mathrm{C}}\right\}=\left[T_{\mathrm{Cl}}\right]\left\{F_{1}\right\}
$$

Then the data is transferred from the CAD to the target disciplines.

$$
\left\{F_{2}\right\}=\left[T_{2 C}\right]\left\{F_{C}\right\}
$$

The advantage of this approach is that only $\left[T_{C 1}\right]$ and $\left[T_{2 C}\right]$ transformation matrices have to be calculated: $2 n$ matrices instead of $\left(n^{2}-n\right)$.

\section{Transformation Matrices}

It is possible to reformulate the existing methods in terms of transformation matrices. This section presents reformulations of two existing alternative methods.

\section{NURBS-Based Interface ${ }^{14}$}

The NURBS-based process has two steps. In the first step, the discipline model is mapped to the CAD model. Then in the second step, a NURBS representation is used to fit the data.

Most CAD systems provide tools to save a CAD model as a NURBS representation, which then can be used for the mapping step. This section contains a brief overview of NURBS representation; readers should consult Refs. 14 and 22 for more detailed discussion. A NURBS surface, $\bar{R}(u, v)$, can be represented as

$$
\bar{R}(u, v)=\frac{\sum_{i}^{l} \sum_{j}^{\prime} W_{i j} B_{i, p}(u) B_{j, q}(v) \bar{P}_{i j}}{\sum_{i}^{l} \sum_{j}^{\prime} W_{i j} B_{i, p}(u) B_{j, q}(v)}
$$

where the $\bar{P}_{i j}$ are the locations of NURBS control points, the $W_{i j}$ coefficients are the weights, $u$ and $v$ are the parameters, $I$ and $J$ are the numbers of control points in $u$ and $v$ directions, and $B_{i, p}(u)$ and $B_{j, q}(v)$ are the B-spline basis functions of degrees $p$ and $q$ respectively. This equation can be written in a compact form by combining the weights and basis functions into a single term as

$$
\begin{gathered}
\bar{R}(u, v)=\sum_{n}^{l * J} C_{n}(u, v) \bar{P}_{n}=[C][\bar{P}], \\
\text { where } n=i+I^{*}(j-1), \quad \text { and } \\
C_{n}(u, v)=C_{i j}(u, v)=\frac{W_{i j} B_{i, p}(u) B_{j, q}(v)}{\sum_{k}^{l} \sum_{l}^{J} W_{k l} B_{k, p}(u) B_{l, q}(v)}
\end{gathered}
$$

Typically the data from the source discipline, $\bar{F}_{1}=\left\{\bar{f}_{1}, \bar{f}_{2}, \ldots, \bar{f}_{m}, \ldots\right\}^{T}$, defines loads or deflection vectors at a discrete set of points $\bar{r}_{m}$. In the first step we project each source grid point $\bar{r}_{m}$ onto the CAD NURBS surfaces and find the appropriate surface and the associated parameters, $u_{m}$ and $v_{m}$. The projection reduces the number of independent variables from three, $\bar{f}_{m}\left(x_{m}, y_{m}, z_{m}\right)$, to two, $\bar{f}_{m}\left(u_{m}, v_{m}\right)$. The independent variables $\left(u_{m}, v_{m}\right)$ are the parametric coordinates of the point $\bar{r}_{m}$ on the NURBS surface. This information may be available from the grid generation process. If not, the grid points can be projected onto the original NURBS surface (see Ref. 23).

A NURBS surface is then fitted through the data to form 


$$
\tilde{f}_{1}(u, v)=[C\}\left\{\bar{F}_{\mathrm{C}}\right\}
$$

The fitted surface is based on a least-squares approximation (see Refs. 14-16) that minimizes the approximation error. The weights and the knot vectors of the CAD NURBS surface can be used, or the user can specify weights and knot vectors. The degrees of NURBS approximation, $p$ and $q$, and the knot vector distribution affect the smoothness of the least-squares representation. The minimization error $E$ can be written as

$$
\begin{gathered}
E^{2}=\left\|\left\{\bar{F}_{1}\right\}-\tilde{f}_{1}(u, v)\right\|^{2}= \\
\left\{\left\{\bar{F}_{1}\right\}-[C]\left\{\bar{F}_{\mathrm{C}}\right\}\right\}^{T}\left\{\left\{\bar{F}_{1}\right\}-[C]\left\{\bar{F}_{\mathrm{C}}\right\}\right\}
\end{gathered}
$$

The least-squares form of the above equation can be expressed in a matrix form as

$$
\left\{\bar{F}_{\mathrm{C}}\right\}=\left[T_{C 1}\right]\left\{\bar{F}_{1}\right\}
$$

where

$$
\left[T_{C l}\right]=\left[C^{T} C\right]^{-1}[C]^{T}
$$

A generic high-speed civil transport (HSCT) geometry was used to demonstrate the algorithm ${ }^{14}$. This geometry was made of three surfaces: fuselage, inboard wing, and outboard wing. Figure 3 shows the original (undeflected) NURBS surfaces, the deflected CSM grid, and the deflected NURBS surfaces. To test the limits of the deflection-transfer algorithm, the CSM grid had a large and unrealistic deflection.

Because the CSM grid is generally coarser than the CFD surface grid, the interpolation approach for deflection transfer may produce a discontinuous CFD surface grid. However, the NURBS-based approach maintained the smoothness of the geometry for deflection transfer. This is a major advantage for the NURBS based approach. On the other hand, for transferring the integrated quantities, the process in its present form did not guarantee conservation of forces and moments. Further studies need to be performed to determine whether the advantages from the smoothness of the NURBS approach outweigh the lack of conservation.

\section{Interpolation-Based Algorithm}

We followed the algorithms proposed in Refs. 18 and 21 . The first step is to map each source and target grid point to the CAD model as described earlier, and map the CAD model to the source and target disciplines. We used a discrete representation of the CAD model, which is readily available from most $C A D$ systems. Each source and target point was mapped to the CAD model, and the parametric coordinates $(u, v)$ of the source and target grid points were determined from the mapping process. The second step is to transfer the data from the source discipline to the target discipline. In this step, the parametric coordinates were used to transfer the data to the CAD model:

$$
\bar{F}_{\mathrm{C}}=\sum_{i} N_{i}\left(u_{i_{1}}, v_{i_{1}}\right) \bar{F}_{i_{1}}
$$

where the term $N_{i}$ was the FE shape function. This equation was applied for each source grid point. The resulting set of equations was assembled into

$$
\left\{\bar{F}_{\mathrm{C}}\right\}=\left[T_{C 1}\right\}\left\{\bar{F}_{1}\right\}
$$

The elements of the transformation matrix were made of the FE shape functions. A similar process was used to transfer the data from the CAD model to the target discipline. As demonstrated by other researchers, ${ }^{78}$ the IBA was very effective in transferring the scalar and vector quantities.

The original ${ }^{18}$ and the modified IBA ${ }^{21}$ were used to transfer the aerodynamics data for a blendedwing body and an HSCT model. Figure 4 shows the result of transferring sensitivity derivative data from a CFD grid to a CSM grid for an HSCT model using the original algorithm. The data is the sensitivity derivative of pressure with respect to the leading-edge sweep angle.

Figure 5 shows the result of transferring the pressure distribution for a blended-wing body using the original algorithm. The figure shows the pressure contours on the CFD grid and the pressure contours transferred from the CFD grid to the CAD model. It also shows the pressure contours transferred from the CAD model to the CSM grid. As expected, the original IBA ${ }^{18}$ did not maintained the conservation of forces and moments. The error for the integrated forces 
was approximately $5 \%$. This error would be higher when the models have dissimilar levels of geometry details.

For transfer of the integrated quantities, the modified IBA had a slightly different implementation (see Ref. 21), which conserves forces and moments. Figure 6 shows the load vectors on the CFD grid and the load vectors transferred from the CFD grid to the CAD model. It also shows the load vectors transferred from the CAD model to the CSM grid. As expected ${ }^{21}$, the transfer process maintained the conservation of forces and moments within the machine's accuracy. This process can also be used to transfer deflections from a CSM grid to a CFD grid.

Figure 7 shows the result of transferring the aeroelastic deflections from a CSM grid to a CAD model and then to the CFD grid. The topleft figure shows the deflected CSM grid. The deflection is transferred from the CSM grid to the CAD model, as shown in Fig. 7 (middle-left figure). Then the deflection is transferred from the CAD model to the CFD grid (bottom-left figure). The right portion of the figure shows the deflected CSM and CFD grids, and they are right on top of each other. A closer look at the Fig. 7 demonstrates the benefit of the three-

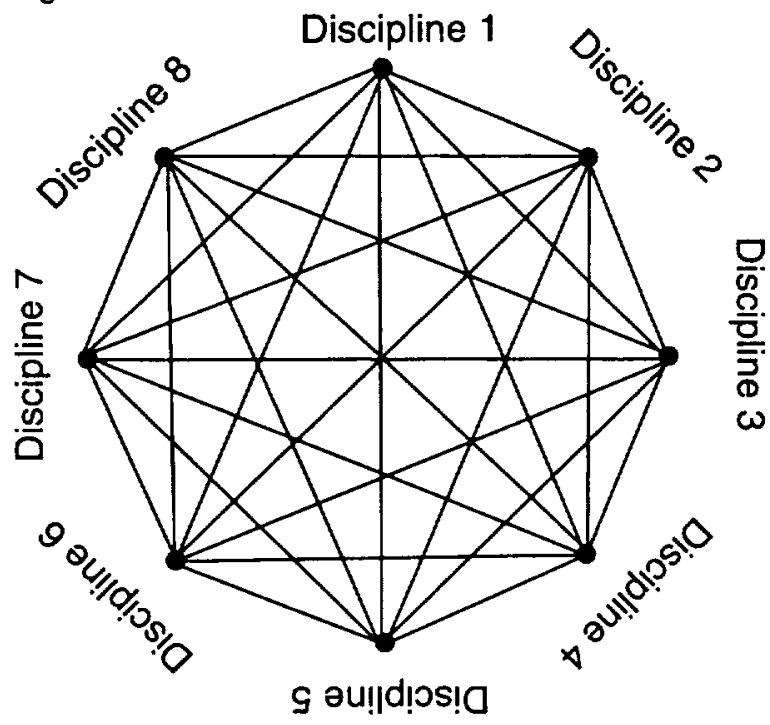

Fig. 1 Multidisciplinary interactions. dimensional data transfer. The winglet is not merely shifted up-it has followed the CSM deflection without neglecting the $x$ and $y$ components of the deflection. The traditional data transfer will only include the vertical displacement ( $z$ coordinates).

\section{Summary}

We have presented a unified approach for transferring information for a multidisciplinary application with $n$ disciplines. This method has two essential ingredients. First, the data transfer process between two disciplines was modeled by a transformation matrix. Second, the CAD model with consistent geometry was used to reduce the number of interactions from $\left(n^{2}-n\right)$ to $2 n$. This unified approach was specially tailored for application environments where the geometry is created and managed through a CAD system. Results were presented for a high-speed civil transport and a blendedwing body. The approach provides a framework where data transfer among disciplines can be accomplished consistently, and potentially with a high degree of automation. Comparisons between the NURBS and IBA using CAD and transformation matrices are continuing.

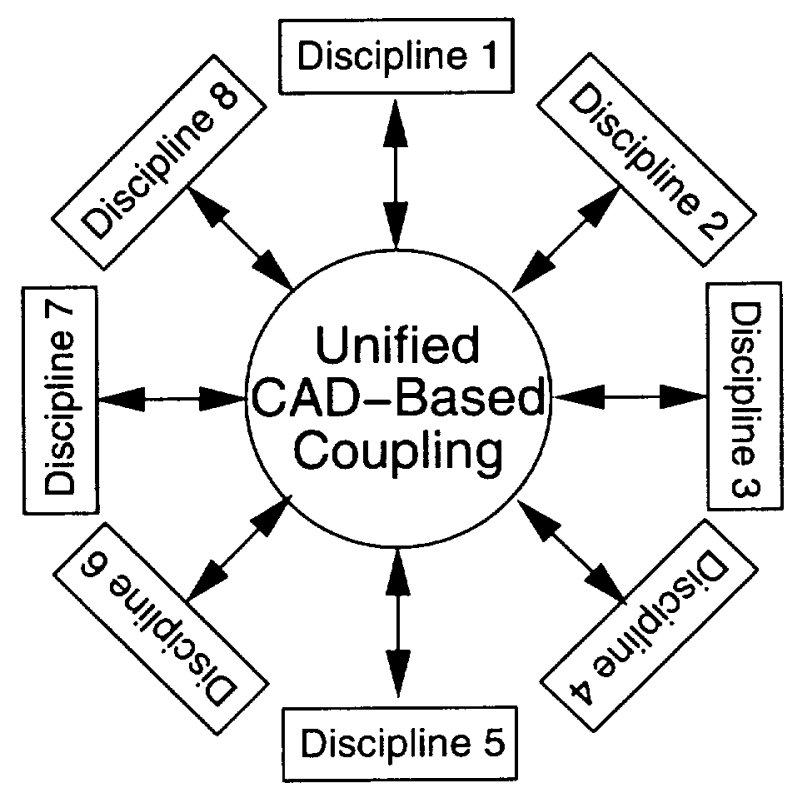

Fig. 2 Unified multidisciplinary interactions. 


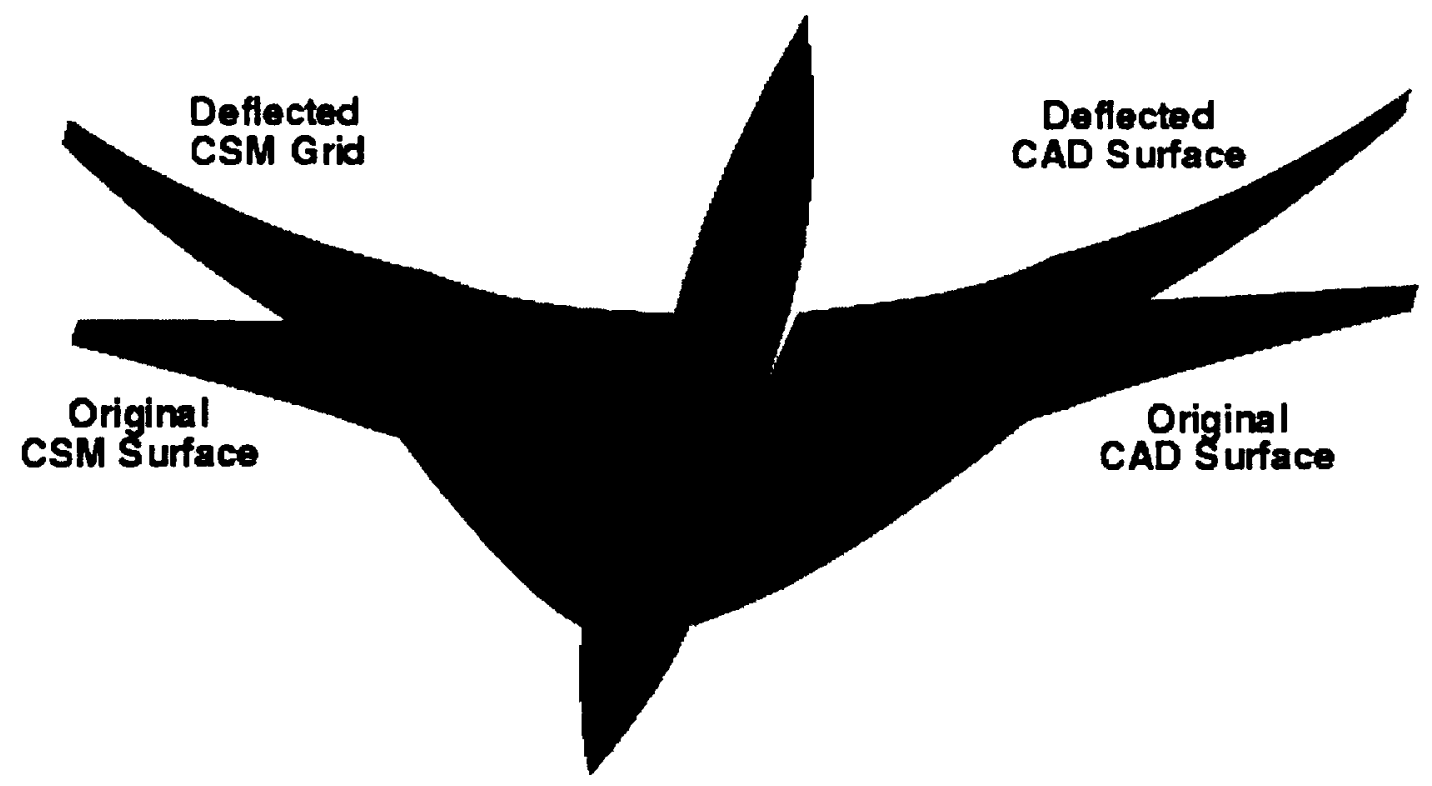

Fig. 3 Aeroelastic deflection transferred from CSM grid to a CAD model.

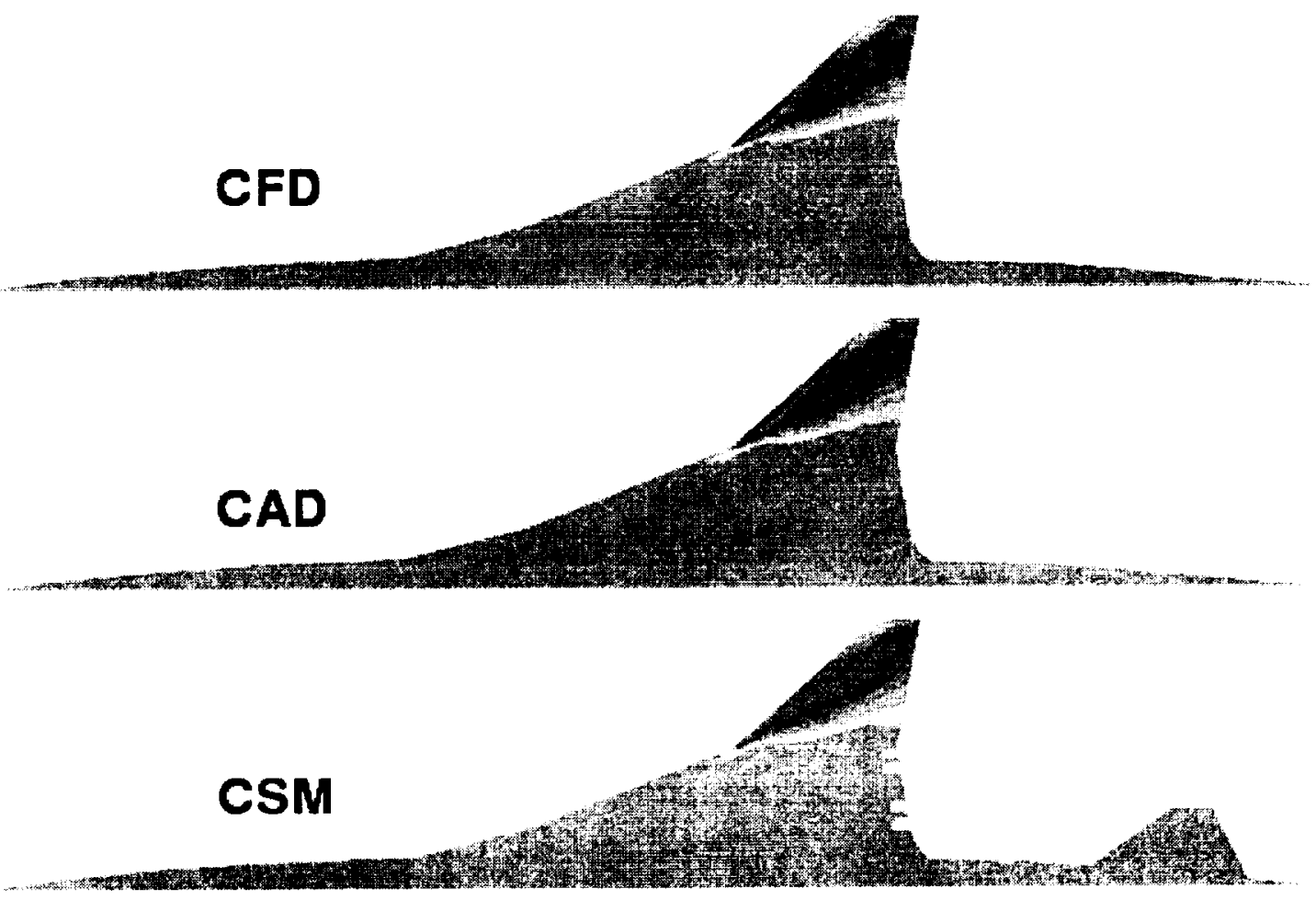

Fig. 4 Transfer of sensitivity derivative from CFD grid to CSM grid.

American Institute of Aeronautics and Astronautics 


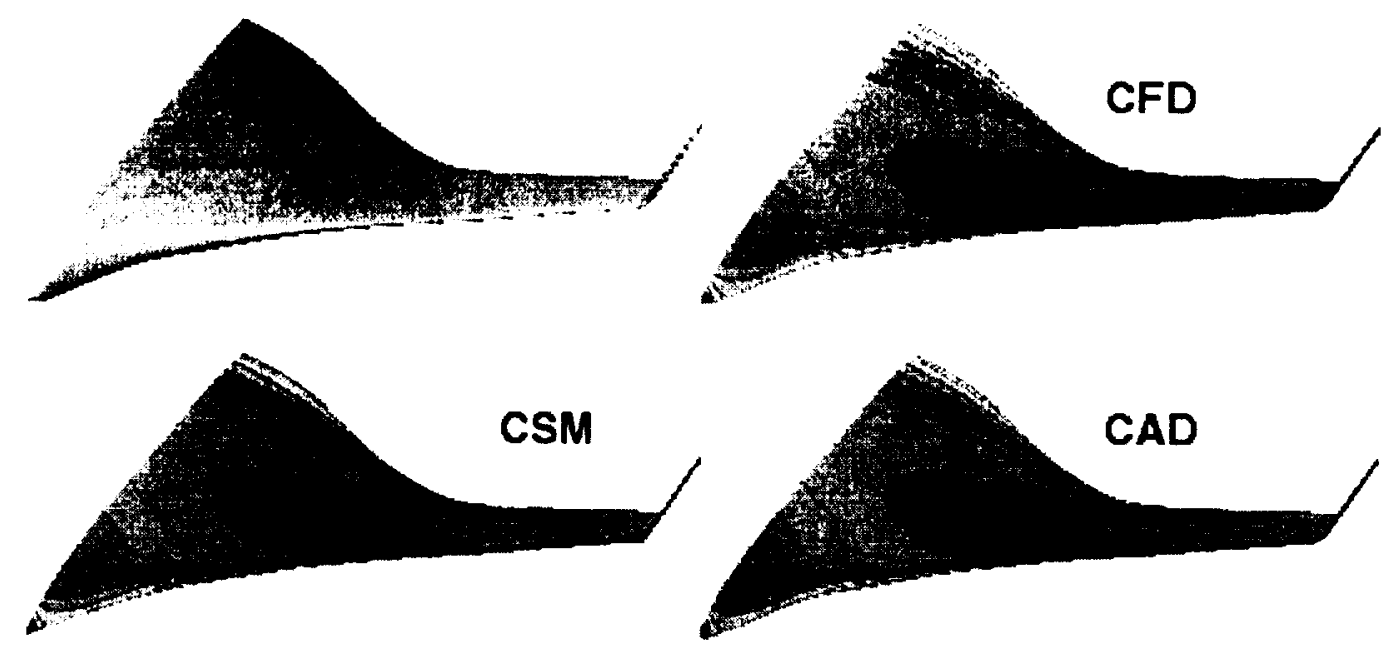

Fig. 5 Pressure interpolation from CFD grid to CSM grid.
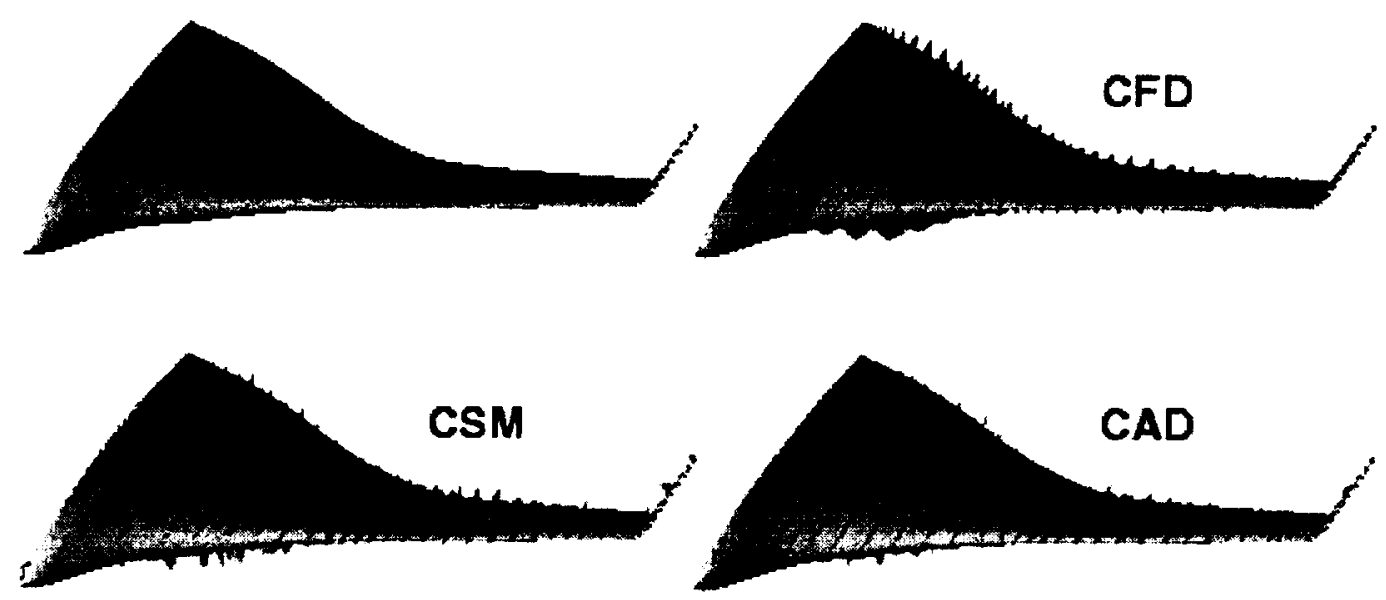

Fig. 6 Transfer of aerodynamic load vectors from CFD grid to CSM grid. 


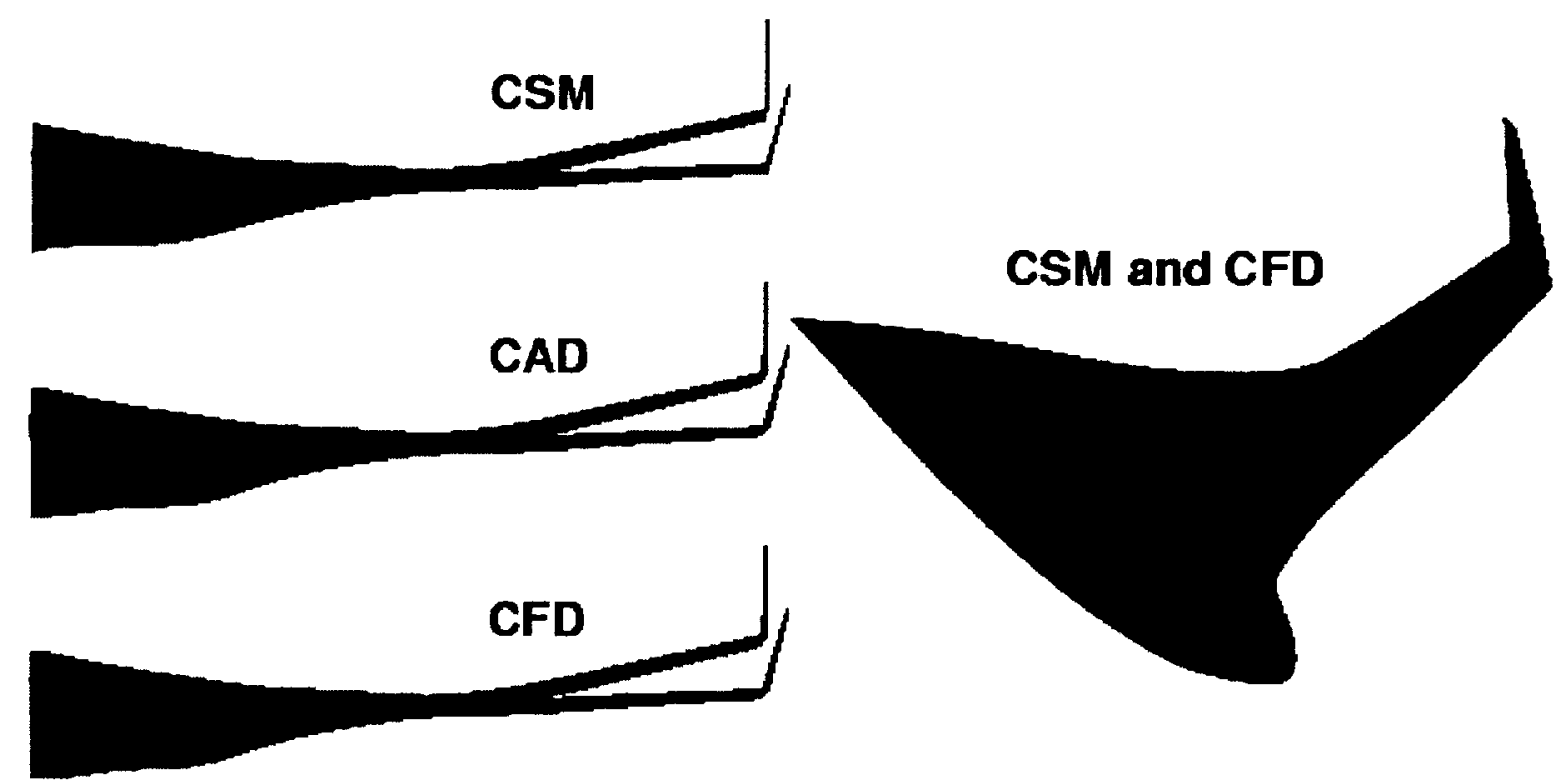

Fig. 7 Transfer of aeroelastic deflection from CSM grid CFD grid.

\section{References}

${ }^{1}$ Dusto, A. R., "A Method for Predicting the Stability Characteristics of an Elastic Airplane, FLEXSTAB Theoretical Description," NASA CR-114,712, Oct. 1974.

2 Petiau, C. and Brun, S., "Trends in Aeroelastic Analysis of Combat Aircraft," AGARD, AD-P005855, Aug. 1987.

${ }^{3}$ Nicot, Ph., and Petiau, C., "Aeroelastic Analysis Using Finite Element Models," European Forum for Aeroelasticity and Structural Dynamics," Aachen, Germany, 1989.

${ }^{4}$ Tzong, G., Chen, H. H., Chang, K. C., Wu, T., and Cebeci, T., "A General Method for Calculating AeroStructure Interaction on Aircraft Configurations," AIAA Paper 96-3982, Sept. 1996.

${ }^{5}$ Kapania, R. K., and Bhardwaj, M., "Aeroelastic Analysis of Modern Complex Wings," AIAA Paper 964011, Sept. 1996.

${ }^{6}$ Brown, S. A., "Displacement Extrapolations for CFD+CSM Aeroelastic Analysis," AIAA Paper 97-1090, Apr. 1997.

${ }^{7}$ Cebral, J. R., and Löhner, R., "Fluid-Structure Coupling: Extensions and Improvements," AIAA Paper 97-0858, Jan. 1997.

${ }^{8}$ Hounjet, M. H. L., and Meijer, J. J., "Evaluation of Elastomechanical and Aerodynamic Data Transfer Methods for Non-Planar Configurations in Computational Aeroelastic Analysis," The Proceedings of the 
International Forum on Aeroelasticity and Structural Dynamics, 1995.

${ }^{9}$ Smith, M. J., Hodges, D. H., and Cesnik, C. E. S., "An Evaluation of Computational Algorithms to Interface Between CFD and CSD Methodologies," Wright-Patterson Laboratory, WL-TR-96-3055, Nov. 1995.

${ }^{10}$ Smith, M. J., Hodges, D., and Cesnik, C., "Fluids and Structures Interface Toolkit (FASIT), Version 1.0," Georgia Tech Research Institute, Report GTRI A-9812-200, Atlanta, GA, 1996.

${ }^{11}$ Harder, R. L., and Desmarais, R. N., "Interpolation Using Surface Splines," AIAA Journal, Vol. 9, No. 2, 1972, pp. 189-191.

${ }^{12}$ Clutter, E. G., "A NURBS Based Interface Definition for Fluid-Structure Interactions Studies," Master's thesis, Department of Aerospace Engineering, Mississippi State University, Mississippi, Dec. 1997.

${ }^{13}$ Send, Wolfgang, "Coupling of Fluid and Structure for Transport Aircraft Wings," International Forum on Aeroelasticity and Structural Dynamics, CEAS/AIAA/ICASE/NASA Langley, Williamsburg, VA, June, 1999. (Not included in the bounded volume)

${ }^{14}$ Samareh, J. A., "Use Of CAD Geometry in MDO," The 6th AIAAUSAF/NASAISSMO Symposium on Multidisciplinary Analysis and Optimization, AIAA-96-3991, Bellevue, WA, Sept. 1996, pp. 88-98.

${ }^{15}$ Hayes, J. G., and Halliday, J., "The Least-Squares Fitting of Cubic Spline Surfaces to General Data Sets," Journal of Institute of Applied Mathematics and Applications, Vol. 14, No. 1, 197, pp. 89-1034.

${ }^{16}$ Dierckx, P., Curve and Surface Fitting with Splines, Clarendon Press, New York, 1993.

${ }^{17}$ Cook, R. D., Malkus, D. S., and Plesha, M. E., Concepts and Applications of Finite Element Analysis, $3^{\text {rd }}$ ed., John Wiley \& Sons, New York, 1989.

${ }^{18}$ Maman, N., and Farhat, C., "Matching Fluid and Structure Meshes for Aeroelastic Computations: A Parallel Approach," Computers and Structures, Vol. 54, No. 4, 1995, pp. 779-785.

${ }^{19}$ Murti, V., and Valliappan, S., "Numerical Inverse Isoparametric Mapping in Remeshing and Nodal Quality Contouring," Computers and Structures, Vol. 22, No. 6, 1986 pp. 1011-1021.

20 Cebral, J. R., and Löhner, R., "Conservative Load Projection and Tracking for Fluid-Structure Problems," AIAA Journal, Vol. 34, No. 4, 1997, pp. 68-692.

${ }^{21}$ Farhat, C., Lesoinne, M., and LeTallec, P., "Load and Motion Transfer Algorithms for Fluid/Structure Interaction Problems with Non-Matching Discrete Interface: Momentum and Energy Conservation, Optimal Discretization and Application to Aeroelasticity," Computer Methods and Applied Mechanical Engineering, Vol. 157, No. 1, 1998, pp. 95-114.

${ }^{22}$ Farin, G., Curves and Surfaces for Computer Aided Geometric Design, Academic Press, San Diego, 1990.

${ }^{23}$ Samareh, J. A., "Unstructured Grid on NURBS Surfaces," AIAA Paper 93-3454, Aug. 1993. 